Relations industrielles

Industrial Relations

\title{
LAMONTAGNE, Maurice, Le Fédéralisme canadien, 1954. (Québec: Les Presses Universitaires Laval, 298 pages, \$2.50).
}

\section{Yves Dubé}

Volume 9, numéro 3, juin 1954

URI : https://id.erudit.org/iderudit/1022885ar

DOI : https://doi.org/10.7202/1022885ar

Aller au sommaire du numéro

Éditeur(s)

Département des relations industrielles de l’Université Laval

ISSN

0034-379X (imprimé)

1703-8138 (numérique)

Découvrir la revue

Citer ce compte rendu

Dubé, Y. (1954). Compte rendu de [LAMONTAGNE, Maurice, Le Fédéralisme canadien, 1954. (Québec: Les Presses Universitaires Laval, 298 pages, \$2.50).] Relations industrielles / Industrial Relations, 9(3), 303-304.

https://doi.org/10.7202/1022885ar

Tous droits réservés (C Département des relations industrielles de l’Université Laval, 1954
Ce document est protégé par la loi sur le droit d'auteur. L’utilisation des services d'Érudit (y compris la reproduction) est assujettie à sa politique d'utilisation que vous pouvez consulter en ligne.

https://apropos.erudit.org/fr/usagers/politique-dutilisation/ 
lyse distincte des opinions du « rankand-file 》 et des officiers.

A cause de la valeur des méthodes d'enquête utilisées, des limites et réserves que les deux savants professeurs posent eux-mêmes sur la valeur et la portée de leur travail, il semble évident que les conclusions auxquelles ils en arrivent représentent une expression assez juste de l'opinion extrêmement divisée des unionistes américains quant à la participation à la politique. Certes qu'un semblable «gallup poll 》 auprès des membres éclairerait singulièrement les syndicats canadiens et leurs chefs.

\section{Jacques Archambault}

LAMONTAGne, Maurice, Le Fédéralisme canadien, 1954. (Québec: Les Presses Universitaires Laval, 298 pages, $\$ 2.50)$.

Voici un ouvrage qui arrive à un moment particulièrement opportun dans lhistoire de la Confédération canadienne. Dans la fièvre du débat qui s'est єngagé depuis quelques anées autour de la répartition des fonctions et des sources de taxation entre le gouvernement central et ceux des provinces, on a semblé oublier, particulièrement dans la province de Québec, les objectifs économiques, sociaux et même culturels, qui doivent être à la base des préoccupations de tout Etat moderne. Au Canada francais, ce problème de la répartition des fonctions et des pouvoirs de taxation a été présenté presqu'exclusivement comme un des aspects du problème plus vaste de l'équilibre entre les deux groupes ethniques principaux du pays. Pourtant, comme le démontre le professeur Lamontagne, la considération franche et sereine de ces objectifs pourrait soit amener un terme au débat, soit, encore mieux, l'orienter dans des voies plus fructueuses et ceci à l'avantage de la population canadienne et plus spécificuement de celle de la province de Québec.

Le travail se divise en deux parties. La première est surtout historique. Elle cherche à mettre en lumière les évènements politiques, économiques et sociaux qui se sont passés depuis le milieu du siècle dernier et qui ont été d'abord la cause de la Confédération, ensuite de la réorientation périodique de cette dernière et qui nous ont conduits finalement à la situation présente. Le professeur Lamontagne est ce qu'on a convenu d'appeler, dans les cercles académiques, un \& fonctionaliste 》, c'està-dire qu'il cherche à expliquer les changements dans la structure politique de notre pays par les faits auxquels ce dernier $\mathrm{a}$ eu à faire face. On doit avouer que l'histoire du Canada depuis 1867 justifie amplement son attitude. Cette façon de voir le fait conclure à une distinction nette entre le fédéralisme des premières années et celui d'aujourd'hui, qu'il appelle respectivement l'ancien et le nouveau fédéralisme, distinction qu'il rappellera continuellement par la suite. Le lecteur trouvera également très intéressant le récit des faits qui ont conduit à l'apogée de l'autonomie provinciale et ensuite à son déclin.

La deuxième partie est plutôt théorique. Il s'agit d'évaluer le «nouveau fédéralisme canadien » à la lumière des derniers développements de la science économique. Le professeur Lamontagne établit d'abord que notre régime écoromique continuera d'être caractérisé par la prédominance de l'entreprise privée. Dans un pays comme le nôtre où les ressources naturelles sont abondantes, les incitations à investir sont nombreuses. Laisser l'investissement aux entrepreneurs privés n'est, en somme, qu'assurer le développement le plus rapide de notre pays. Cependant. l'entreprise privée et l'industrialisation entrânent néressairement le chômage - qu'il soit cyclique, structurel ou saisonnier - et l'insécurité sociale, pour la population laborieuse comme pour la population inactive. L'Etat doit intervenir pour remédier à ces maux qui dépassent les pouvoirs de l'initiative privée et c'est cette nouvelle fonction de l'Etat nui distingue nettomont le nouveau fédéralisme de l'ancien. L'auteur étudie ici une multitude de problèmes. Le chômage est sans doute le plus important mais il traite aussi avec soin des impôts sur le revenu et sur la consommation, de l'assurance-chômage, de la négociation collective, du monopole, du problème agricole, etc...

A l'occasion de chaque ordre de problème, il montre qu'on ne saurait songer à faire une répartition complète et définitive des responsabilités entre les r'ifférents gouvernements. Ceci est d'ailleurs sa thèse générale. Même s'il 
est en faveur d'une délimitation des fonctions quand cela est possible, il démontre qu'elle peut être impossible dans la plupart des cas. Le chômage luimême ne saurait être de juridiction strict'ement fédérale. Le professeur Lamonfagne n'est ni à priori autonomiste, ni à priori centralisateur. Il est « fonctionaliste ». Si nous voulons éviter une plus grande centralisation et un changement radical de la Constitution, il faudra que chaque unité de gouvernement contribue d'une façon éclairée et dans la mesure de ses pouvoirs à la solution conjointe des problèmes qui confrontent aujourd'hui le citoyen canaàien.

L'auteur réfute ceux qui veulent placer le débat uniquement sur le plan nationaliste. Il établit que les Canadiens français se sont toujours cherché des boucs émissaires sur qui jeter le blâme de leurs insuccès. Ces boucs émissaires ont été successivement les Anglais, les Juifs et finalement le gouvernement dOttawa. Il est temps que nous envisagions 《notre question nationale 》 sous son angle positif, qui est l'enrichissement et le rayonnement de la culture française au Canada et que surtout nous prenions conscience des réalités économiques et sociales de notre pays.

Ceux qui ont franchement à coeur d'être informés sur la nature des problèmes politiques qui se posent aujourd'hui au Canada trouveront dans ce volume une abondante matière à réflexion. L'auteur lui-même en a dit que c'était une invitation à la réflexion en même temps qu'un plaidoyer pour la compréhension. Nous le recommandons spécialement aux chefs d'entreprises et de mouvements ouvriers comme étant le travail le plus important publié depuis longtemps sur la question constitutionnelle.

Yves DubÉ

MAITRON. Jean, Le Syndicalisme révolutionnaire Paul Delesalle, 1 vol. $174 \mathrm{p}$. Editions Ouvrières, 1019, rue St-Denis, Montréal, prix: $\$ 2.50$.

Cet ouvrage est la biographie d'un homme qui a incarné une forme de syndicalisme qui, en France, à une époque eut un certain retentissement: le svndicalisme révolutionnaire. Bien qu'en raison de nos principes, il nous soit im- possible d'accepter et la doctrine et la technique d'action de Paul Delesalle, le personnage, comme homme, vaut la peine d'être connu et mérite d'être apprécié pour sa sincérité. Nous conseillons d'une manière spéciale la lecture de cet ouvrage aux observateurs superficiels de la réalité syndicale. Ils y apprendront beaucoup de choses dont la moindre n'est pas de distinguer entre le véritable syndicalisme révolutionnaire et le syndicalisme que l'on rencontre en notre pays. Ils constateront encore que le syndicalisme révolutionnaire authentique rejettait toute action politique. Enfin, d'une façon indirecte, ils y verront pourquoi les circonstances historiques n'ont pas laissé aux catholiques de France d'autre issue que de créer un syndicalisme chrétien. Cet ouvrage est, en somme, un chapitre de la grande histoire du mouvement ouvrier.

\section{GÉrard Dion}

MARCY, G. RoBinot - La C.G.T., Force Ouvrière dans Revue de l'Action Populaire, janvier 1954, pp. 49-64.

Excellent article dans lequel l'auteur retrace l'évolution de la C.G.T. - F.O. depuis sa naissance jusqu'à nos jours.

PHELPS, ORME W. - Community Recognition of Union Leaders - Industrial and Labor Relations Review, Volume 7. Number 3, April 1954. po. 419-434. Issued quarterly by New York State School of Industrial and Labor Relations at Cornell University, Ithaca, New York, $\$ 5.00$ a year $-\$ 1.50$ a copy.

Le professeur Phelps enseigne les relations du travail au Claremont Men's College (U.S.A.). Il a écrit son article alors qu'il était boursier du Social Science Research Council et du Twentieth Century Fund. Est-ce que les chefs ouvriers américains possèdent un statut social en accord avec leur autorité et leur responsabilité? Telle est la question à laquelle l'auteur veut répondre. A cette fin, il examine successivement de quelle façon sont récompensés les chefs ouvriers au point de vue salaires et par rapport aux titres honorifiques qui leur sont conférés. Trouve-t-on plusieurs des 350 chefs ouvriers améri- 